\title{
Ulcerative colitis in the South African Bantu
}

\author{
J. D. SOBEL AND L. SCHAMROTH \\ From Baragwanath Hospital and the University of Witwatersrand, Johannesburg
}

SUMMARY Two cases of ulcerative colitis in the Bantu are reported. One patient presented with fulminant acute colitis with toxic dilatation of the colon and massive rectal haemorrhage, the second with chronic, continuous diarrhoea for over twenty years, culminating ultimately with carcinoma of the colon.

The aetiology of ulcerative colitis is discussed, together with the problems of specific diagnosis encountered in a population with a high incidence of parasitic infestation and infective diarrhoea.

It is considered that the sparseness of reports and apparent rarity of this disease in the Bantu may reflect a failure of detection rather than a true infrequent occurrence. The natural incidence of the disease may be on the increase.

Ulcerative colitis has long been considered a rare condition in the Bantu (Negro) population. The infrequency of this disease in tropical and subtropical Africa was commented upon in a geographical survey conducted in 1962 (British Medical Journal, Editorial, 1962). Billinghurst in 1964 described the first three cases of idiopathic ulcerative colitis in Africans treated at Mulago Hospital, Uganda. Pillay subsequently reported four cases diagnosed in Durban in 1964 and in the same centre Powell and Wilmot (1966) have recently encountered additional cases of ulcerative colitis both postdysenteric and idiopathic in origin.

This report describes two further cases of ulcerative colitis of undetermined aetiology, which occurred in two adult Bantu women seen at Baragwanath Hospital during the first three months of 1969.

\section{Case 1}

A 28-year-old Sotho woman was admitted in January 1969 with a three-week history of Received for publication 26 January 1970. dysentery. She passed four to 12 stools per day, which consisted mainly of fresh blood and mucus. Mild vomiting had been present during the first week of the illness but subsequently disappeared. There was minimal abdominal pain. Her admission had been precipitated by marked weakness and one syncopal episode. It is noteworthy that at no time in the previous few years had she been troubled by diarrhoea or any gastrointestinal upset; nor was there any relevant family history of a diarrhoeal disorder. She was resident in Johannesburg and had not travelled to any subtropical region.

Examination revealed a distressed, shocked young woman who was cold and clammy with marked conjunctival pallor. She was restless, inattentive, and mildly confused. The pulse rate was 130 per minute and of poor volume. The blood pressure was $60 / 40 \mathrm{~mm} \mathrm{Hg}$ and there was marked clinical acidosis. The abdomen was not distended and was minimally tender. Proctoscopic examination demonstrated an oedematus and granular rectal mucosa.

Laboratory investigations revealed the following: the haemoglobin count was $6.6 \mathrm{~g} \%$; the red cells showed hypochromia and target cell $\overline{\bar{c}}$. formation; the white blood count was $42,000 \risingdotseq$ 
$\mathrm{c} / \mathrm{mm}$, with toxic granulations of the neutrophils and a moderate shift to the left; blood urea $112 \mathrm{mg} / 100 \mathrm{ml}$; serum potassium $4.0 \mathrm{~m}$-equiv/l; serum sodium $110 \mathrm{~m}$-equiv/l; serum chlorides $83 \mathrm{~m}$-equiv/l; and $\mathrm{CO}_{2}$ combining power 12 m-equiv/litre. No amoebae were detected on numerous microscopic examinations of the stools.

Because of the local prevalence of amoebic colitis and the rarity of ulcerative colitis in the African a therapeutic trial of metronidazole $(800$ $\mathrm{mg}$ ) was begun quite empirically in spite of the lack of any confirmatory evidence for this diagnosis. Supportive therapy and intravenous tetracycline was also given.

Repeated attempts to isolate amoeba during the ensuing week were to no avail, whilst stool and blood cultures were likewise consistently negative. The Widal test for typhoid fever was also negative. Sigmoidoscopy revealed a hyperaemic oedematous and hypertrophied mucosa, which bled easily when touched. Rectal biopsy showed foci of ulceration with chronic inflammation of the mucosa. The histological features were consistent with nonspecific ulcerative colitis.

There was no response to the therapy and the dysentery and severe constitutional signs persisted. Radiology showed a dilated transverse colon, a toxic megacolon (Fig.). On electrocardiography there was a marked sinus tachycardia, 100/min, with nonspecific T-wave changes. Metronidazole was stopped and the patient was given 1 grain of dihydroemetine daily, $250 \mathrm{mg}$ chloroquine every six hours, as well as intravenous chloramphenicol $(500 \mathrm{mg})$ every six hours.

No improvement was noted during the following week and she had two episodes of massive rectal haemorrhage which required transfusion and vigorous resuscitation. The marked constitutional features persisted and intensive replacement of intravenous fluid was required. Hypocalcaemia (3.2 m-equiv/l) and hypomagnesaemia $(1.1 \mathrm{~m}$-equiv/l) with resultant tetany and tremor were identified. Both states responded to replacement therapy.

A second rectal biopsy revealed severe colitis and ulceration and again failed to demonstrate the presence of amoebae. It was then decided to treat the patient for idiopathic nonspecific ulcerative colitis, and she was given $100 \mathrm{mg}$ hydrocortisone hemisuccinate sodium intravenously every six hours as well as hydrocortisone hemisuccinate sodium rectal drips 100 $\mathrm{mg}$ twice daily. There was a gradual but definite improvement in the diarrhoea, but the tachycardia, pyrexia, and leucocytosis persisted, as did the toxic megacolon. Colectomy was accordingly considered, but was postponed because of the recent administration of emetine and the presence of the persistent severe tachycardia. Furthermore the sigmoidoscopic appearance of the rectum and of the sigmoid colon suggested a

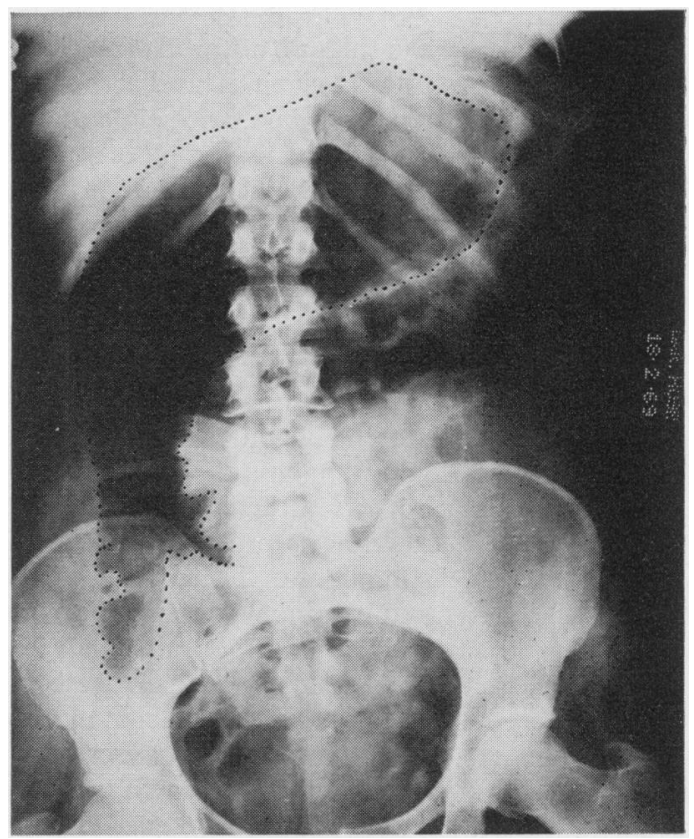

Fig. Case 1. Radiograph of the abdomen showing toxic dilatation of the ascending and transverse colon.

possible improvement. Sulphasalazine $2 \mathrm{~g}$ orally every six hours was commenced.

The improvement continued during the course of the next fortnight; there was no further significant bleeding and the stool frequency decreased to three or four daily. Parenteral fluid therapy was no longer required and the electrolyte status was well maintained. Surgery was again contemplated because of the persistent tachycardia $(140 / \mathrm{min})$, leucocytosis, and colonic dilatation. The patient, however, felt better, she was alert and interested in her surroundings and reluctant, at this stage, to consent to surgery. Conservative therapy was therefore continued: the hydrocortisone was replaced by $60 \mathrm{mg}$ prednisone daily and prednisolone 21-phosphate retention enemata.

Improvement continued and one week later she was ambulant. The patient then complained of tiredness and some minutes later was found dead in bed.

Necropsy revealed innumerable shallow ulcers 0.5 to $1 \mathrm{~cm}$ in diameter, extending along the entire length of the colon. The rectal mucosa appeared macroscopically to be sloughing. There was slight dilatation of both ventricles of the heart and the myocardium was flabby. No evidence of pulmonary embolism was found.

\section{Case 2}

This 54-year-old, pure Sotho woman developed 
dysentery in 1948. She had been investigated at a mission hospital in 1949, but no specific therapy was given. The diarrhoea continued over the next 21 years, with eight to 10 watery stools containing mucus and blood daily. She had lost $110 \mathrm{lb}$ during this period. Arthritic pain in the hands, knees, and spine had been present for some years.

An increase in the severity of symptoms necessitated an admission to Baragwanath Hospital in May 1967. Barium enema demonstrated a rigid colon with loss of the haustral pattern. Sigmoidoscopy revealed diffusely granular, haemorrhagic mucosa. Rectal biopsy showed irregular polypoid proliferation of the mucosa, fibrosis of the lamina propria, hypertrophy of the muscularis mucosa, and fatty infiltration of the submucosa. There was a marked plasma cell and lymphocytic infiltration in the lamina propria and submucosa and small crypt abscesses were present. The features were consistent with the diagnosis of nonspecific ulcerative colitis.

Cortisone was prescribed both orally and rectally but for some unknown reason, surgery was not undertaken. The diarrhoea persisted but her present admission was prompted by symptoms of vomiting, abdominal distension, and colicky abdominal pain as well as profound weakness. Diarrhoea was still present.

Examination revealed a moderately obese, distressed middle-aged woman with profound muscular weakness. The abdomen was distended, hypersonant to percussion, with a few, isolated, tinkling bowel sounds. Significant muscular wasting was present, predominantly affecting the face, shoulder, and hip girdle.

The haemoglobin was found to be $12.2 \mathrm{~g} / 100$ $\mathrm{ml}$; the white blood count $7,200 \mathrm{c} / \mathrm{mm}$; the blood urea $72 \mathrm{mg} / 100 \mathrm{ml}$; serum sodium 135 m-equiv/l; serum potassium $2.7 \mathrm{~m}$-equiv/l; serum chlorides 98 m-equiv/l; $\mathrm{CO}_{2}$ combining power was $15 \mathrm{~m}$-equiv/l; calcium, phosphorus, serum protein, and liver function tests were normal. Radiology of the abdomen showed gross dilatation of the small intestine with numerous fluid levels present.

Sigmoidoscopy to $25 \mathrm{~cm}$ again confirmed the presence of oedematous, granular mucosa, and many bleeding sites but no ulcers were seen.

Laparotomy was performed after rehydration and therapy for potassium replacement. A large constricting ring carcinoma of the transverse colon was found with gross dilation of the proximal colon and small bowel. The carcinoma was mobile, with minimal evidence of microscopic adjacent infiltration. No enlarged glands were found. The liver appeared to be normal. After small bowel decompression, a loop ileostomy was performed.

\section{Discussion}

There has been little documented evidence of ulcerative colitis existing in subtropical and tropical regions; the disease is considered to be essentially one of temperate zones (Melrose, 1955). However, recent reports (Billinghurst, 1964; Pillay, 1964; Powell and Wilmot, 1966; Chuttani, Nigan, Sama, Dhanda, and Gupta, 1967) have suggested that the disease is by no means as uncommon as previously considered, and that the incidence may be on the increase in these regions (Billinghurst and Welchman, 1966).

Johannesburg is situated at an altitude of 6,000 feet and enjoys an extremely mild, temperate climate. The Bantu population for the most part constitute a recently urbanized group who have been rapidly transformed from a relatively primitive rural society to a pseudo-sophisticated 'western' form of life. Ulcerative colitis in the Bantu has not previously been described in this area in spite of the normal incidence of the disease encountered in the white inhabitants. It would thus appear that there is an ethnic rather than a geographical limiting factor. The Bantu population served by Baragwanath Hospital has a high incidence of parasitic infestation and acute dystentery is a very common occurrence. It may well be that many mild and moderate attacks of ulcerative colitis, both idiopathic and postdysenteric, are undetected amongst the great mass of patients with infective diarrhoea (Billinghurst and Welchman, 1966). This lack of diagnositic precision in the face of the large number of cases, together with, in general, a poor long-term follow up of the patients, may well explain the past failure to detect ulcerative colitis in the Bantu. On the other hand a genuine increase in the incidence of the disease may well have occurred simultaneously with the rapid social reorientation of the urbanized Bantu population. 'Westernization' has brought with it completely new dietary regimens, as well as significant physical and emotional stress. These drastic environmental changes may be responsible for a true increase in the occurrence of idiopathic ulcerative colitis.

Such consideration must, however, be seen in the light of other recent advances in the pathogenesis of idiopathic ulcerative colitis. The evidence suggests an immunological basis for the diseased colon, probably at a cellular immune level. It is of interest, therefore, that Zoutendyk (personal communication, 1969) has found autoimmune disease to be much less common in the Bantu people.

In neither of our two cases was there any evidence of the typical personality traits, nor was the disorder related to known psychological stress. Both patients consumed the predominant maize diet typical of the urbanized African.

The first case presented as an acute, fulminating ulcerative colitis of undetermined aetiology, in which numerous attempts (stool microscopy, and mucosal histology) to isolate the amoebae, both during life and at necropsy,were negative. It is not 
possible to state emphatically that amoebiasis can be completely excluded since the amoebic agar gel diffusion test was not done. However, amoebae are almost invariably found in the acute phase. The absence of amoebae in acute life-threatening colitis should alert one to the possibility of idiopathic ulcerative colitis irrespective of the population group concerned. It is nevertheless clear that fulminant, life-threatening ulcerative colitis with toxic dilatation of the colon and massive rectal haemorrhage does occur in the Bantu, and treatment should, during the acute phase, follow the same principles as outlined for the idiopathic variety (Truelove, 1968; Goligher, 1968).

The second case had a chronic continuous course, quite closely simulating the presentation of chronic idiopathic ulcerative colitis. The negative finding of the amoebic agar-gel diffusion test at a late stage is of no value in excluding the diagnosis of an initial amoebic colitis. However, the extremely protracted course followed by this patient does not resemble the postdysenteric variety, which tends to improve with time. Furthermore, the development of a carcinoma of the colon after 23 years of disease is not unexpected and has not been seen to develop in the postdysenteric variety of colitis (Powell and Wilmot, 1966). It is thus evident that although ulcerative colitis has in the past appeared to be rare in the African, evidence is now accumulating of a not infrequent occurrence both in its mild and severe form.

We should like to thank the Photographic Department, Department of Medicine, University of the Witwatersrand, for the photographic reproduction.

\section{References}

Billinghurst, J. R. (1964). Ulcerative colitis in the African. Rep. of 11 th Ann. Meeting of Ass. of Physicians of East Africa. Brit. med. J., 2, 117.

Billinghurst, J. R., and Welchman, J. M. (1966). Idiopathic ulcerative colitis in the African: a report of four cases. Brit. med. J., 1, 211-213.

Chuttani, H. K., Nigam, S. P., Sama, S. K., Dhanda, P. C., and Gupta, P. S. (1967). Ulcerative colitis in the tropics. Brit. med. J., 4,204-207.

British Medical Journal (1962). Editorial. Geograpical destruction of ulcerative colitis. Brit. med. J., 4, 36 .

Goligher, J. C. (1968). Surgical treatment of ulcerative colitis. Brit. med. J., 1, 671-674.

Melrose, A. G. (1955). The geographical incidence of chronic ulcerative colitis in Britain. Gastroenterology, 29, 1055-1060.

Pillay, V. K. G. (1964). Ulcerative colitis in the African. Brit. med. J., $2,689$.

Powell, S. J., and Wilmot, A. J. (1966). Ulcerative postdysenteric colitis. Gut, 7, 438-443.

Truelove, S. C. (1968). Medical management of ulcerative colitis. Brit. med. J., 2, 539-542.

Zoutendyk, A. (1969). Personal communication.

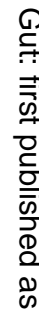

